\title{
Ueber die Doppeltangenten einer ebenen Kurve vierter Ordnung, inbesondere über den Aronholdschen Satz.
}

von E. BoDewig (Köln a. Rh., Germania).

\section{EDUARDO STUDY IN MEMIORIAM}

In den Berliner Monatsberichten von 1864 gab Aronhold über die Doppeltangenten einer ebenen $C_{4}$ zwei beruhmte Sätzo bekannt, die seit dieser Zeit seinen Namen tragen. Insbesondere enthält der zweite Satz - dass (mit einer gewissen Einschränkung) sieben beliebige Geraden immer als ein System von 7 Doppeltangenten einer $C_{4}$ aufgefasst werden kömen, aus denen man die übrigen 21 Doppeltangenten rational finden kann - eine der schönsten Aufgaben der ganzen Feometrie. Die Aronholdschen Beweise dieser beiden Sätze gingen unverändert in alle späteren Lehrbücher über. Erst Study hatte starke Bedenken gegen den zweiten dieser Sätze. Worauf sich seine Vermutung stützte, ist mir nicht bekannt. Jedenfalls veranlasste er mich noch kurz vor seinem Tode, mich mit dem Problem zu beschäftigen. Zu meinem eigenen Erstaunen fand ich seine Vermutung bestätigt. Es ist mir daher eine Pflicht der Dankbarkeit, diese Arbeit dem Andenken meines hochverehrten Lehrers zu widmen.

Die Darstellung benutzt durchgängig die symbolische Methode, die ja auch den Arbeiten Studys und Arouholds am nächsten kommt. Gleichzeitig erhalten dadurch die Resultate eine gefälligere und symmetrische Form. Die Theorie der Doppeltangenten wurde nochmal von Anfang an behandelt, jedoch nur dort, wo sich Abkürzungen gegenüber der üblichen Darstellung ergaben. Zugrunde gelegt wurde der Gedankengang, wie ihu Weber in seinem Lehrbuch entwickelt, da dieser bei dem Problem selbst bleibt und nicht mit Dingen, wie Flächen dritter Ordnung, arbeitet, die dem Problem im Anfange fremd sind. Doch konnte die Webersche Darstellung in zwei Punkten wesentlich abgekürzt und durch das Ausscheiden von fremden Elementen natürlicher gestaltet werden, uämlich an den Stellen (11)-(13) des Textes, wo es sich um die Gradbestimmung der die Berührungspunkte ausschneidenden Kurve handelt, und bei den syzygetischen Tripeh. Bei dem Aronholdschen Satz selbst finden 
sich in (23) zwei Ausdrücke für die $\lambda$ sowie die Beziehung (24), die bei Weber fehlen und die doch das Ganze so sehr vereinfachen. Dadurch folgt (2õa) und (26a) auf einfache Weise, während bei Aronhold wieder umständliche Rechnungen nötig sind. Aehnlich (33) und (33a). Gleichung (42) und (43) führen dann das Problem zu Ende. Es ergibt sich, dass für den zweiten Salz die Aronholdsche Bedingung allein nicht ausveicht, sondern dass noch eine weitere Bedingung hinzutreten muss.

\section{Die Berechnung der Zahl der Doppeltangenten.}

Die Kurve sei $(a x)^{4}=0$. Setze ich $\xi+\lambda \eta$ anstelle von $x$, so ist

$(a, \xi+\lambda \eta)^{4}=(a \xi)^{4}+4 \lambda(a \xi)^{3}(a \eta)+6 \lambda^{2}(a \xi)^{2}(a \eta)^{2}+4 \lambda^{3}(a \xi)(a \eta)^{3}+\lambda^{4}(a \eta)^{4}$.

Icb nehme nun an, die beiden ersten Glieder in obigem Ausdruck verschwänden, d. h. es sei

$$
\begin{gathered}
(a \xi)^{4}=0: \text { d. h. } \xi \text { sei ein Kurvenpunkt } \\
(a \xi)^{3}(a \eta)=0: \text { d. h. } \eta \text { liege auf der Tangente in } \xi .
\end{gathered}
$$

Es bleibt demnach für die weiteren Schnittpunkte der Tangente mit der Kurve die Gleichung übrig:

$$
6(a \xi)^{2}(a \eta)^{2}+4 \lambda(a \xi)(a \eta)^{3}+\lambda^{2}(a \eta)^{4}=0 .
$$

Soll die Tangente Doppeltangente sein, so muss die Diskriminante

$$
\Delta(\stackrel{2}{\xi}, \quad \stackrel{6}{\eta})=3(a \xi)^{2}(a \eta)^{2}(b \eta)^{4}-2(a \xi)(a \eta)^{3}(b \xi)(b \eta)^{3}=0 .
$$

(Die Gradzahlen 2 und 6 in $\xi$ und $\eta$ sind darübergesetzt worden). Diese Gleichung soll umgewandelt werden in eine Gleichung in $\xi$ allein.

Für $\eta$ gilt nun Gleichung (2); man kann also setzen

$$
(\eta u) \equiv(a \xi)^{3}(\alpha k u) \text { identisch in } u \text {, }
$$

wobei $k$ unbestimmt ist. Geometrisch heisst dies: Man wählt denjenigen Punkt $\eta$ aus, in welchem dje Tangente in $\xi$ von der Geraden $(k x)=0$ geschnitten wird; $k$ ist wie gesagt willkürlich, unterliegt aber doch der Beschränkung, dass

$$
(k \xi) \neq 0
$$

d. h. $k$ darf nicht durch unseren Kurvenpunkt $\xi$ gehen, da sonst $\xi$ mit $\eta$ zusammenfiele.

Trägt man obigen Ausdruck für $\eta$ in die Diskriminante ein, so erhält man

$$
\Delta\left(\xi, a k(a \xi)^{3}\right) \equiv D(\xi \stackrel{20}{20}, \stackrel{6}{k}),
$$


d. h. man erhält eine Funktion, die in $\xi$ vom Grade 20 und in $k$ vom Grade 6 ist. Das Verschwinden von $D$ ist die notwendige und hinreichende Bedingung dafür, dass $\xi$ der Berührungspunkt einer Doppeltangente ist, Ausserdem muss aber noch $(k \xi) \neq 0$. Verstehe ich nun unter $\xi$ einen der vier Schnittpunkte von $k$ mit der Kurve, so folgt $\xi=\eta$, da ja $\eta$ der Schnittpunkt von $k$ mit der Tangente in $\xi$ ist. Wegen $\xi=\eta$ wird dann $\Delta(\xi, \xi)=0$, da $\xi$ auf der Kurve liegt. Demnach ist auch für die umgeformte Diskriminante $D(\xi, k)=0$, d. h. die Kurve 20. Grades $D(x, k)=0$ läuft für alle $k$ durch die vier Schnittpunkte von $k$ mit der Kurve. Diese vier Schnittpunkte sind unserem Problem fremd, sie müssen daher eliminiert werden.

Man nimmt dazu statt $\eta$ einen anderen Punkt $\zeta$ der Tangente, den man etwa durch die Gerade $m$ mit der Gleichung $(m x)=0$ auf der Tangente $(a \xi)^{3}(\alpha x)=0$ ausschneidet. Dann ist also $(\zeta u) \equiv(a \xi)^{3}(a m u)$ identisch in $u$. Man sucht den Wert, den jetzt die Diskriminante $\Delta(\xi, \zeta)$ annimmt. Da $\zeta$ auf der Tangente liegt, so gibt es gewisse Zahlen $\lambda$, $\mu$, so dass $\zeta=\lambda \xi+\mu \eta$. Wegen $(a \xi)^{4}=(a \xi)^{3}(a \eta)=0$ gehen jetzt die in der Diskriminante $\Delta$ auftretenden Ausdrücke in folgende Werte über:

Danach wird

$$
\begin{aligned}
& (a \xi)^{2}(a \zeta)^{2}=\mu^{2}(a \xi)^{2}(a \eta)^{2} \\
& (b \zeta)^{4}=6 \lambda^{2} \mu^{2}(b \xi)^{2}(b \eta)^{2}+4 \lambda \mu^{3}(b \xi)(b \eta)^{3}+\mu^{4}(b \eta)^{4} \\
& (a \xi)(a \zeta)^{3}=3 \lambda \mu^{2}(a \xi)^{2}(a \eta)^{2}+\mu^{3}(a \xi)(a \eta)^{3}
\end{aligned}
$$

$$
\Delta(\xi, \xi)=\mu^{B} \cdot \Delta(\xi, \eta)
$$

natürlich nur, wenn $\xi$ ein Kurvenpunkt und $\eta$ und $\zeta$ Punkte der Tangente in $\xi$ sind. Für diese Werte von $\xi$ nnd $\eta$ ist demnach $\Delta(\xi, \eta)$ eine Kovariante vom Gewichte 6 gegentiber Transformationen der Form $\xi^{\prime}=\xi, \eta^{\prime}=\lambda \xi+\mu \eta$. Führt man statt $\eta$ und $\zeta$ die Grôssen $k$ und $m$ ein, so geht jedesmal die Diskriminante $\Delta$ in die zugehörige $D$ über, so dass auch

$$
D(\xi, \stackrel{6}{m})=\mu^{6} \cdot D(\xi, \stackrel{6}{k}) \text {. }
$$

Man darf vermuten, dass, wemn man durch die sechste Potenz eines Linearausdruckes in $m$ dividiert, man einen von $m$ freien Ausdruck erhält. Es handelt sich zunächst darum, die Grösse $\mu$ aus $\mathrm{k}$ und $\mathrm{m}$ allein au bestimmen. Nun ist

$$
\begin{aligned}
(m \zeta) & =\lambda(m \xi)+\mu(m \eta)=0 \\
(k \zeta) & =(a \xi)^{3}(a m k)=\lambda(k \xi), \quad \text { da } \quad(k \eta)=0 \\
(m \eta) & =(a \xi)^{3}(a k m)=-\lambda(k \xi) .
\end{aligned}
$$


Jetzt ist also

Demmach ist

$$
\begin{gathered}
\mu(m \eta)=-\lambda(m \xi)=-\lambda \mu(h \xi), \mathrm{d} . \mathrm{h} . \\
\mu=(m \xi):(k \xi) .
\end{gathered}
$$

$$
D(\xi, m):(m \xi)^{6}=D(\xi, k):(k \xi)^{i},
$$

d. h. der Ausdruck

$$
D(\xi, m):(m \xi)^{b} \text { ist von } m \text { unabhängig. }
$$

Da die Beziehung (8) für alle Kurvenpunkte $\xi$ gilt, so enthält die Kurve 26. Grades

$$
(k x)^{6} \cdot D(x, n)-(m x)^{6} \cdot D(x, k)=0
$$

die $C_{4}$ als Teilkurve. Es ist demnach

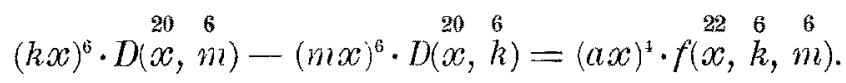

Wir wollen den Bat der Funktion $f$ näher bestimmen. In (11) steht liuks eine Zerlegung nach dem Modul $(k x)^{6},(m x)^{6}$. Angenommen, $f$ lasse bei der Zerlegung einen Rest $R$, so müsste $R \cdot(a x)^{4} \equiv 0$ modd $(k x)^{6},(m x)^{6}$. Man zerlege num $(a x)^{4} \operatorname{modd}(k x),(m x)$. Sind $(k x)=0,(m x)=0$ so gewählt, dass sie sich nicht auf der $C_{4}$ schneiden, so bleibt auch bei dieser Zerlegung ein Rest $r$, der mit $R$ multipliziert $\equiv \equiv 0$ modd $(k x)^{6},(m x)^{6}$ wäre. Demnach ist $R=0$. Ich setze daher

$$
f(x, \stackrel{6}{h,} \stackrel{6}{m})=(k x)^{6} \cdot f_{1}(x, k, m)-(m x)^{6} \cdot f_{2}(x, k, m) .
$$

$f_{1}$ hat nun in $k$ den Grad Null, da ja $f$ in $k$ den Grad 6 hat; ähnlich $f_{z}$. Wir schreiben daher besser

$$
\begin{aligned}
& \begin{array}{llll}
22 & 6 & 6 \\
16 & 6 & 16 & 6
\end{array} \\
& f(x, k, m)=(k x)^{6} \cdot f_{1}(x, m)-(m x)^{6} \cdot f_{2}(x, k) .
\end{aligned}
$$

Setzen wir dies in (11) ein, so erhalten wir

$$
(k x)^{6}\left[D(x, m)-(a x)^{4} \cdot f_{1}(x, m)\right]=(m x)^{6}\left[D(x, k)-(a x)^{4} \cdot f_{2}(x, k)\right] .
$$

Es ist demnach die Klammer links durch $(m x)^{6}$ teilbar, diejenige rechts durch $(k x)^{6}$. Die Quotienten können aber nicht mehr von $k$ bzw. $m$ abhängen, da ja die Klammern in $m$ bzw. $k$ jedesmal nur den Grad 6 haben: Also ist

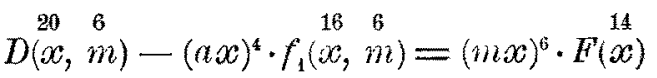

$$
\begin{aligned}
& D(x, k)-(a x)^{4} \cdot f_{2}(x, k)=(k x)^{6} \cdot F(x) \text {. }
\end{aligned}
$$

Diese ganze Funktion $\mathrm{F}(\mathrm{x})$, die von keinem Parameter mehr abhängt, verschwindet nun ebenfalls für alle Berührungspunkte der Doppeltangenten, 
wie sich ja aus jeder der beiden Gleichnngen (13).ergibt. Bringe ich sie also mit der $C_{4}$ zum Schnitt, so erhalte ich die Höchstzahl der Berührungspunkte der Doppeltangenten, nämlich 56. Demnach ist die Höchstzahl der Doppeltangenten selbst gleich 28. Da es andererseits Kurven gibt, wie die Kleinsche Kurve, welche wirklich 28 Doppeltangenten haben, so hat jede $\mathrm{C}_{4} 28$ Doppeltangenten.

\section{Die Steinerschen Komplexe.}

Sei $t_{1}$ eine Doppeltangente (kurz: $D T$ ). Dann lässt sich zerlegen:

$$
(a x)^{4}=\left(t_{1} x\right) \cdot(A x)^{3}-(B x)^{4} .
$$

Da aber die $C_{4}$, mit $t_{1}$ zum Schnitt gebracht, zwei Doppelpunkte ergibt, so muss $(B x)^{4}$ zerfallen in ein Quadrat:

Setzt man jedoch

$$
(a x)^{4}=\left(l_{1} x\right)(A x)^{3}-\left[(B x)^{2}\right]^{2} .
$$

$$
\begin{aligned}
(A x)^{3}+2(\lambda x)(B x)^{2}+(\lambda x)^{2}\left(t_{1} x\right) & =\left(A_{\lambda} x\right)^{3} \\
(B x)^{2}+(\lambda x)\left(t_{1} x\right) & =\left(B_{\lambda} x\right)^{2},
\end{aligned}
$$

wo $(\lambda x)$ oine beliebige Linearform ist, so gilt auch jetzt noch die Gleichung:

$$
(a x)^{4}=\left(t_{1} x\right)\left(A_{\lambda} x\right)^{3}-\left[\left(B_{2} x\right)^{2}\right]^{2} .
$$

$u_{1}$ sei eine zweite $D T$. Ihre Berührungspunkte seien $p$ und $q .(\lambda x)$ soll jetzt so gewählt werden, dass $\left(B_{3} x\right)^{2}=0$ durch $p$ und $q$ läuft, so dass demnach

$$
\left(A_{\lambda} p\right)^{3}=\left(A_{\lambda} q\right)^{3}=\left(B_{\lambda} p\right)^{2}=\left(B_{\lambda} q\right)^{2}=0 .
$$

Bilde ich jetzt die Tangente in $n$ :

$$
\begin{aligned}
(a p)^{3}(a x)=3\left(t_{1} p\right)\left(A_{\lambda} p\right)^{2}\left(A_{\lambda} x\right)+\left(t_{1} x\right)\left(A_{\lambda} p\right)^{3}- & \\
& -4\left(B_{\lambda} p\right)^{2} \cdot\left(B_{\lambda} p\right)\left(B_{\lambda} x\right)=3\left(t_{1} p\right)\left(A_{\lambda} p\right)^{2}\left(A_{\lambda} x\right)=0
\end{aligned}
$$

so sieht unan, dass diese Tangente $u_{1}$ auch Tangente von $\left(A_{\lambda} x\right)^{3}=0$ ist, und ZWar in $p$ und $q$, dass sie also $D T$ ist. $\left(A_{2} x\right)^{3}$ zerfällt daher in $\left(u_{1} x\right)(C x)^{2}$. (Dabei muss $\left(t_{1} p\right) \neq 0$, da sonst $C_{4}$ einen Doppelpunkt hätte). Wir haben also jetzt:

Setzt man

$$
(a x)^{4}=\left(t_{1} x\right)\left(u_{1} x\right)(C x)^{2}-\left[(B x)^{2}\right]^{2} .
$$

$$
\begin{aligned}
& (B x)^{2}+\mu\left(t_{1} x\right)\left(u_{1} x\right)=\left(B_{\mu} x\right)^{2} \\
& (C x)^{2}+2 \mu(B x)^{2}+\mu^{2}\left(t_{1} x\right)\left(u_{1} x\right)=\left(C_{\mu} x\right)^{2}
\end{aligned}
$$

so ist auch dann noch

$$
(a x)^{4}=\left(t_{1} x\right)\left(u_{1} x\right)\left(C_{\mu} x\right)^{2}-\left[\left(B_{\mu} x\right)^{2}\right]^{2} .
$$


Bestimmen wir $\mu$ so, dass $\left(C_{\mu} x\right)^{2}$ zerfällt, so erhalten wir eine Gleichung vom Grade 5 in $\mu$. Danach haben wir die Zerlegungen für $\left(C_{\mu} x\right)^{2}$ :

Also ist auch

$$
\left(t_{2} x\right)\left(u_{2} x\right) ; \quad\left(t_{3} x\right)\left(u_{3} x\right) ; \ldots . . ;\left(t_{6} x\right)\left(u_{6} x\right) .
$$

$$
(a x)^{\dagger}=\left(t_{1} x\right)\left(u_{1} x\right)\left(t_{k} x\right)\left(u_{k} x\right)-\left[\left(B_{k} x\right)^{2}\right]^{2}, \quad k=2, \ldots, 6 \text {; }
$$

Daraus ergibt sich auf die bei Weber-Fricke beschriebene Weise schlieslich die Darstellung

$$
\begin{aligned}
(a x)^{4} & =2\left(l_{2} x\right)\left(u_{2} x\right)\left(l_{3} x\right)\left(u_{3} x\right)+2\left(t_{3} x\right)\left(u_{3} x\right)\left(l_{1} x\right)\left(u_{1} x\right)+ \\
& +2\left(t_{1} x\right)\left(u_{1} x\right)\left(t_{2} x\right)\left(u_{2} x\right)-\left(t_{1} x\right)^{2}\left(u_{1} x\right)^{2}-\left(t_{2} x\right)^{2}\left(u_{2} x\right)^{2}- \\
& -\left(t_{3} x\right)^{2}\left(u_{3} x\right)^{2} .
\end{aligned}
$$

Schneidet man die $C_{4}$ mit $t_{k}$ oder $u_{k}$, so folgt aus (14), dass man jedesmal zwei Doppelpunkte erhält, $d . h$. auch die $t_{k}$ und $u_{k}$ sind $D T$. Auf jedem $\left(B_{k} x\right)^{2}=0$ liegen demnach die 8 Berührungspunkte von $4 D T: t_{1}, u_{1}, t_{k}, u_{k}$. Ein solches System von 6 DT-Parren heisst ein Steinerscher Komplex. In Verbindung mit (15) folgt in bekannter Weise, dass die Berührungspunkte je zweier Paare eines Steinerschen Komplexes auf einem Kegelschuitt liegen.

\section{Syzygetische Tripel.}

Daraus ergibt sich auf bekamte Weise der Begriff des syzygetischen und azygetischen Tripels. Ein syzygetisches Tripel liegt immer vor, wenn aus einem Steinerschen Komplex ein Paar und noch eine DT herausgegriffen wird. Es fragt sich, ob dies die einzige Art dieser Tripel ist, oder ob auch ein Tripel, das drei verschiedenen Parren eines Steinerschen Komplexes angehört, syzygetisch sein kann. Wir wählen dazu das Tripel $\left(t_{1} x\right),\left(t_{2} x\right),\left(t_{3} x\right)$. Aus der Darstellung:

$$
\left.(a x)^{4}=4\left(t_{1} x\right)\left(u_{1} x\right)\left(t_{2} x\right)\left(u_{2} x\right)--\left[\left(t_{1} x\right)\left(u_{1} x\right)+\left(t_{2} x\right) u_{2} x\right)-\left(t_{3} x\right)\left(u_{3} x\right)\right]^{2}
$$

ergibt sich, dass die Berührungspunkte von $t_{1}, t_{2}, u_{1}$ auf dem Kegelschnitt

$$
\left(t_{1} x\right)\left(u_{1} x\right)+\left(t_{2} x\right)\left(u_{2} x\right)-\left(t_{3} x\right)\left(u_{3} x\right)=0,
$$

die Berührungspunkte von $t_{1}, t_{2}$ allein also auf dem Büschel

$$
\left(t_{1} x\right)\left(u_{1} x\right)+\left(t_{2} x\right)\left(u_{2} x\right)-\left(t_{3} x\right)\left(u_{3} x\right)+\lambda\left(t_{1} x\right)\left(t_{2} x\right)=0,
$$

wo $\lambda$ ein Parameter ist, liegen. Aehnlich liegen die Berührungspunkte von $t_{2}, t_{3}$ auf

$$
\left(t_{2} x\right)\left(u_{2} x\right)+\left(t_{3} x\right)\left(u_{3} x\right)-\left(t_{1} x\right)\left(u_{1} x\right)+\mu\left(t_{2} x\right)\left(t_{3} x\right)=0 .
$$


Da aber beide Büschel kein Element gemeinsam haben, liegen die Berührungspunkte von $t_{1}, t_{2}, t_{3}$ nicht auf einem Kegelschnitt. Das Tripel ist also azygetisch.

Daraus folgt, wiederum auf bekannte Weise, die Existenz der Aronhold'schen Systeme.

Wir kommen dann zu unserem Hauptteil: dem Aronholdschen Satz.

\section{Der Aronholdsche Satz.}

Es sei

$$
t_{1}, t_{2}, t_{3}, t_{4}, t_{5}, t_{6}, t_{7}
$$

ein beliebiges Aronholdsches System. Da jedes aus ihm bildbare Tripel azygetisch ist, so laufen keine drei dieser Tangenten durch einen Punkt, $\mathrm{d} . \mathrm{h}$. jede aus diesen Grössen gebildete Determinante ist von Null verschieden.

Wir nehmen aus (16) der Reihe nach die $D T t_{1}$ bzw. $t_{2}$ bzw. $t_{3}$ heraus und bilden für die übrigen $D T$ jedesmal den zugehörigen Steinerschen Komplex, in dem sie enthalten sind. Nach bekannten Resultaten bekommen wir so, wenn wir statt $t_{i}$ nur $i$, statt $u_{i}$ nur $i^{\prime}$ setzen, die Komplexe:

$$
\begin{array}{lllllllll}
2, & 3^{\prime} & 3,2^{\prime} & 4, u_{41} & 5, u_{51} & 6, u_{61} & 7, u_{71} \\
3,1^{\prime} & 1,3^{\prime} & 4, u_{42} & 5, u_{52} & 6, u_{62} & 7, u_{72} \\
1,2^{\prime} & 2,1^{\prime} & 4, u_{43} & 5, u_{53} & 6, u_{63} & 7, u_{73} .
\end{array}
$$

Dabei bedeutet z. B. $u_{52}$ die in dem durch die Heraushebung von 2 entstehenden Komplex mit 5 gepaarte DT. Aus (17) folgt noch als weiterer Komplex:

$$
1,1^{\prime} \quad 2,2^{\prime} \quad 3,3^{\prime} \quad \ldots .
$$

der mit jedem Komplex aus (17) ein syzygetisches Paar bildet und demnach keine der vier $D T 4,5,6,7$ enthält.

\section{Unser Ziel ist, die $D T 1^{\prime}, 2^{\prime}, 3^{\prime}$ als Funktionen der 1, 2,3 anzugeben.}

Für unsere $C_{4}$ haben wir die Darstellungen:

$$
\begin{aligned}
(a x)^{4} & =4(2 x)\left(2^{\prime} x\right)(3 x)\left(3^{\prime} x\right)-\left[\left(f_{1} x\right)^{2}\right]^{2} \\
& =4(3 x)\left(3^{\prime} x\right)(1 x)\left(1^{\prime} x\right)-\left[\left(f_{2} x\right)^{2}\right]^{2} \\
& =4(1 x)\left(1^{\prime} x\right)(2 x)\left(2^{\prime} x\right)-\left[\left(f_{3} x\right)^{2}\right]^{2} \\
& =4(2 x)\left(3^{\prime} x\right)(4 x)\left(u_{41} x\right)-\left[(k x)^{2}\right]^{2}
\end{aligned}
$$


denn auch letzteres Quadrupel ist syzygetisch. Dabei bedeuten:

$$
\begin{aligned}
& \left(f_{1} x\right)^{2}=-(1 x)\left(1^{\prime} x\right)+(2 x)\left(2^{\prime} x\right)+(3 x)\left(3^{\prime} x\right) \\
& \left(f_{1} x\right)^{2}=+(1 x)\left(1^{\prime} x\right)-(2 x)\left(2^{\prime} x\right)+(3 x)\left(3^{\prime} x\right) \\
& \left(f_{3} x\right)^{2}=+(1 x)\left(1^{\prime} x\right)+(2 x)\left(2^{\prime} x\right)-(3 x)\left(3^{\prime} x\right) .
\end{aligned}
$$

Aus der ersten und letzten Darstellung in (19) folgt:

$$
4(2 x)\left(3^{\prime} x\right)\left[(3 x)\left(2^{\prime} x\right)-(4 x)\left(u_{41} x\right)\right]=\left[\left(f_{1} x\right)^{2}\right]^{2}-\left[(k x)^{2}\right]^{2},
$$

d. h., da $(2 x)\left(3^{\prime} x\right)$ nur in einem der beiden Faktoren der rechten Seite enthalten ist,

Daraus folgt

$$
\begin{gathered}
\left(f_{t} x\right)^{2}-(k x)^{2}=2 \lambda(2 x)\left(3^{\prime} x\right) \\
\lambda\left[\left(f_{1} x\right)^{2}+(k x)^{2}\right]=2\left[(3 x)\left(2^{\prime} x\right)-(4 x)\left(u_{41} x\right)\right] .
\end{gathered}
$$

$$
\lambda\left(f_{1} x\right)^{2}=\lambda^{2}(2 x)\left(3^{\prime} x\right)+(3 x)\left(2^{\prime} x\right)-(4 x)\left(u_{41} x\right) .
$$

Setzen wir für $\left(f_{1} x\right)^{2}$ seinen Wert aus (20) und fügen zu $\lambda$ den Index 1 hinzu (da wir später zyklisch weiter gehen), so ist

$$
\begin{aligned}
(4 x)\left(u_{41} x\right) & =(3 x)\left(2^{\prime} x\right)-\lambda_{1}\left[-(1 x)\left(1^{\prime} x\right)+(2 x)\left(2^{\prime} x\right)+(3 x)\left(3^{\prime} x\right)\right]+\lambda_{1}^{2}(2 x)\left(3^{\prime} x\right) \\
(21)(4 x)\left(u_{42} x\right) & =(1 x)\left(3^{\prime} x\right)-\lambda_{2}\left[-(2 x)\left(2^{\prime} x\right)+(3 x)\left(3^{\prime} x\right)+(1 x)\left(1^{\prime} x\right)\right]+\lambda_{2}^{2}(3 x)\left(1^{\prime} x\right) \\
(4 x)\left(u_{43} x\right) & =(2 x)\left(1^{\prime} x\right)-\lambda_{3}\left[-(3 x)\left(3^{\prime} x\right)+(1 x)\left(1^{\prime} x\right)+(2 x)\left(2^{\prime} x\right)\right]+\lambda_{3}^{2}(1 x)\left(2^{\prime} x\right) .
\end{aligned}
$$

Setzen wir in den beiden letzten Gleichungen $x=41$, multiplizieren sie mit $\lambda_{3}$ bzw. $\lambda_{2}$ und addieren, so erhalten wir, da 1, 1;,4 azygetisch sind, ihre Determinante also nicht verschwindet:

$$
\begin{aligned}
& \lambda_{2} \lambda_{3}=-(124):(134) \\
& \lambda_{3} \lambda_{1}=-(234):(214) \\
& \lambda_{1} \lambda_{2}=-(314):(324),
\end{aligned}
$$

Durch Produktbildung folgt $\left(\lambda_{1} \lambda_{2} \lambda_{3}\right)^{2}=1$, also $\lambda_{1} \lambda_{2} \lambda_{3}= \pm 1$. Also ist

$$
\lambda_{1}=\mp(134):(124), \quad \lambda_{2}=\mp(214):(234), \quad \lambda_{3}=\mp(324):(314) \text {. }
$$

Dabei gelten gleichzeitig die oberen oder unteren Vorzeichen, worüber vorläufig noch Unklarheit herrscht.

Setzen wir in den beiden letzten Gleichungen (21) nunmehr $x=41^{\prime}$, so folgt nach Multiplikation mit $\lambda_{2}$ und $\lambda_{3}$ und Division durch $\left(141^{\prime}\right)$ :

$$
\begin{aligned}
& \lambda_{z} \lambda_{3}=-\left(1^{\prime} 3^{\prime} 4\right):\left(1^{\prime} 2^{\prime} 4\right) \\
& \lambda_{3} \lambda_{1}=-\left(2^{\prime} 1^{\prime} 4\right):\left(2^{\prime} 3^{\prime} 4\right) \\
& \lambda_{1} \lambda_{2}=-\left(3^{\prime} 2^{\prime} 4\right):\left(3^{\prime} 1^{\prime} 4\right)
\end{aligned}
$$


und wieder $\lambda_{1} \lambda_{2} \lambda_{3}= \pm 1$, also

(22a) $\quad \lambda_{1}=\mp\left(1^{\prime} 2^{\prime} 4\right):\left(1^{\prime} 3^{\prime} 4\right), \quad \lambda_{2}=\mp\left(2^{\prime} 3^{\prime} 4\right):\left(2^{\prime} 1^{\prime} 4\right), \quad \lambda_{3}=\mp\left(3^{\prime} 1^{\prime} 4\right):\left(3^{\prime} 2^{\prime} 4\right)$.

Dabei gelten in (22) und (22a) gleichzeitig die Minuszeichen oder Pluszeichen. Setze ich, um die Vorzeichen der $\lambda$ zu bestimmen, in $\left(21_{2}\right)$ wieder $x=41^{\prime}$ und ersetze $\lambda_{z}$ durch seinen Wert aus $(22)$, so erhalte ich

$$
\begin{gathered}
-(214)\left(241^{\prime}\right)\left(2^{\prime} 41^{\prime}\right)+\left(341^{\prime}\right)\left(3^{\prime} 41^{\prime}\right)(214)=\mp\left(141^{\prime}\right)\left(3^{\prime} 41^{\prime}\right)(234) \\
\left(3^{\prime} 41^{\prime}\right)\left[\left(341^{\prime}\right)(214) \pm\left(141^{\prime}\right)(234)\right]=(214)\left(241^{\prime}\right)\left(2^{\prime} 41^{\prime}\right) .
\end{gathered}
$$

Nimmt man in der Klammer das Minuszeichen, so ergibt sich die Klammer als $\left(241^{\prime}\right)(314)$, d. h. es ist

$$
\left(3^{\prime} 41^{\prime}\right)\left(241^{\prime}\right)(314)=(214)\left(241^{\prime}\right)\left(2^{\prime} 41^{\prime}\right) .
$$

Da aber nach (22) und (22a) $\left(1^{\prime} 2^{\prime} 4\right):\left(1^{\prime} 3^{\prime} 4\right)=(134):(124)$, so ist auch die vorhergehende Gleichung richtig, also auch das angenommene Minuszeichen. Es gelten demnach in (22) und (22a) die unteren Vorzeichen:

$$
\begin{aligned}
& \lambda_{1}=(413):(412)=\left(41^{\prime} 2^{\prime}\right):\left(41^{\prime} 3^{\prime}\right) \\
& \lambda_{2}=(421):(423)=\left(42^{\prime} 3^{\prime}\right):\left(42^{\prime} 1^{\prime}\right), \\
& \lambda_{3}=(432):(431)=\left(43^{\prime} 1^{\prime}\right):\left(43^{\prime} 2^{\prime}\right) .
\end{aligned}
$$

Vermöge der hieraus bildbaren Gleichungen $(413)\left(41^{\prime} 3^{\prime}\right)-(412)\left(41^{\prime} 2^{\prime}\right)=0$ usw. folgt

$$
(412)\left(41^{\prime} 2^{\prime}\right)=(423)\left(42^{\prime} 3^{\prime}\right)=(431)\left(43^{\prime} 1^{\prime}\right)=k .
$$

Bei Verwendung der zwischen vier Geraden der Ebene bestehenden Identität (oder, was dasselbe ist, bei Verwendung von Dreieckskoordinaten) erhält man nun die beiden Gleichungen:

$$
\begin{aligned}
& (4 x)=\frac{(423)}{(123)}(1 x)+\frac{(431)}{(231)}(2 x)+\frac{(412)}{(312)}(3 x) \\
& (4 x)=\frac{\left(42^{\prime} 3^{\prime}\right)}{\left(1^{\prime} 2^{\prime} 3^{\prime}\right)}\left(1^{\prime} x\right)+\frac{\left(43^{\prime} 1^{\prime}\right)}{\left(2^{\prime} 3^{\prime} 1^{\prime}\right)}\left(2^{\prime} x\right)+\frac{\left(41^{\prime} 2^{\prime}\right)}{\left(3^{\prime} 1^{\prime} 2^{\prime}\right)}\left(3^{\prime} x\right) .
\end{aligned}
$$

Vormöge (24) gehen aber diese Darstellungen über in:

$$
\begin{aligned}
v \cdot(4 x) & =\frac{\left(1^{\prime} 2^{\prime} 3^{\prime}\right)}{\left(42^{\prime} 3^{\prime}\right)}(1 x)+\frac{\left(2^{\prime} 3^{\prime} 1^{\prime}\right)}{\left(43^{\prime} 1^{\prime}\right)}(2 x)+\frac{\left(3^{\prime} 1^{\prime} 2^{\prime}\right)}{\left(41^{\prime} 2^{\prime}\right)}(3 x) \\
& =v_{1}^{\prime}(1 x)+v_{2}^{\prime}(2 x)+v_{3}^{\prime}(3 x) \\
v \cdot(4 x) & =\frac{(123)}{(423)}\left(1^{\prime} x\right)+\frac{(231)}{(431)}\left(2^{\prime} x\right)+\frac{(312)}{(412)}\left(3^{\prime} x\right) \\
& =v_{1}\left(1^{\prime} x\right)+v_{2}\left(2^{\prime} x\right)+v_{3}\left(3^{\prime} x\right),
\end{aligned}
$$


Wo

$$
k \cdot v=(123)\left(1^{\prime} 2^{\prime} 3^{\prime}\right)
$$

Aus (23) schliessen wir ferner, weil $(421)-\lambda_{2}(423)=0$ usw.:

$$
\begin{aligned}
& (A x) \equiv(1 x)-\lambda_{2}(3 x)=0, \quad(2 x)=0, \quad(4 x)=0 \text { laufen durch einen Punkt } \\
& (B x) \equiv(2 x)-\lambda_{3}(1 x)=0, \quad(3 x)=0, \quad(4 x)=0 \quad \text { » } \quad \text { " } \quad \text { » } \\
& (C x) \equiv(3 x)-\lambda_{1}(2 x)=0, \quad(1 x)=0, \quad(4 x)=0 \quad \text { * } \quad \text { * } \quad \text { * } \\
& \left(A^{\prime} x\right) \equiv\left(1^{\prime} x\right)-\lambda_{3}\left(2^{\prime} x\right)=0, \quad\left(3^{\prime} x\right)=0, \quad(4 x)=0 \quad \text { * } \quad \text { * } \quad \text { » } \quad \text { » } \\
& \left(B^{\prime} x\right) \equiv\left(2^{\prime} x\right)-\lambda_{1}\left(3^{\prime} x\right)=0, \quad\left(1^{\prime} x\right)=0, \quad(4 x)=0 \quad \text { » " " । } \\
& \left(C^{\prime} x\right) \equiv\left(3^{\prime} x\right)-\lambda_{2}\left(1^{\prime} x\right)=0, \quad\left(2^{\prime} x\right)=0, \quad(4 x)=0 \quad \text { * } \quad \text { » } \quad \text { » » }
\end{aligned}
$$

sowie

Dabei ist $A B C$ bzw. $A^{\prime} B^{\prime} C^{\prime}$ das Diagonaldreieck des Vierseits 1234 bzw. 1'2'3'4. Aus (29) folgt, dass es Zahlen $\mu_{1}, p_{1}$ gibt, für die

$$
(A x) \equiv(1 x)-\lambda_{2}(3 x)=\mu_{1}(2 x)+p_{1}(4 x)
$$

Setzt man $x=34$ bzw. 32 , so erhält man hieraus und aus den entsprechenden Beziehungen:

Aehnlich ist

$$
\begin{aligned}
& \mu_{1}=\lambda_{3}^{-1} \\
& \mu_{2}=\lambda_{1}^{-1} \\
& \mu_{3}=\lambda_{2}^{-1}
\end{aligned} \quad p_{i}=v_{i} .
$$

usw. wo

$$
\left(A^{\prime} x\right) \equiv\left(1^{\prime} x\right)-\lambda_{3}\left(2^{\prime} x\right)=\mu_{1}^{\prime}\left(3^{\prime} x\right)+p_{1}^{\prime}(4 x)
$$

$$
\begin{aligned}
& \mu_{1}^{\prime}=\mu_{3}=\lambda_{2}{ }^{-1} \\
& \mu_{2}^{\prime}=\mu_{1}=\lambda_{3}{ }^{-1} \\
& \mu_{3}^{\prime}=\mu_{2}=\lambda_{i}^{-1}
\end{aligned} \quad \rho_{i}^{\prime}=v_{i}^{\prime} .
$$

Ferner ist noch wegen (25a), (26a), (27) und (24)

$$
v_{1} v_{1}^{\prime}=v_{2} v_{2}^{\prime}=v_{3} v_{3}^{\prime}=v_{1}-
$$

Dividiere ich jetzt die Gleichungen (21) der Reihe nach durch $\lambda_{1}, \lambda_{2}, \lambda_{3}$ und addiere, so erhalte ich

$$
\begin{gathered}
(4 x)\left[\left(u_{41} x\right): \lambda_{1}+\left(u_{42} x\right): \lambda_{2}+\left(u_{43} x\right): \lambda_{3}\right]=\left(1^{\prime} x\right)\left[(2 x): \lambda_{3}+\lambda_{2}(3 x)-(1 x)\right]+ \\
+\left(2^{\prime} x\right)\left[(3 x): \lambda_{1}+\lambda_{3}(1 x)-(2 x)\right]+\left(3^{\prime} x\right)\left[(1 x): \lambda_{2}+\lambda_{1}(2 x)-(3 x)\right] .
\end{gathered}
$$

Nun ist aber nach (29a) und (30):

$$
(2 x): \lambda_{3}+\lambda_{2}(3 x)=(1 x)-\nu_{1}(4 x)
$$


Setzt man dies und die ähnlichen Resultate in obige Gleichung ein, so folgt:

$$
\left(u_{41} x\right): \lambda_{1}+\left(u_{42} x\right): \lambda_{2}+\left(u_{43} x\right): \lambda_{3}=-v_{1}\left(1^{\prime} x\right)-v_{2}\left(2^{\prime} x\right)-v_{3}\left(3^{\prime} x\right) .
$$

Setzt man die linke Seite dieser Gleichung gleich Null, so erhält man nach (26a) die Gerade $(4 x)=0 .-$

Dividiert man die Gleichung $\left(21_{1}\right)$ durch $\lambda_{1}$, die zweite durch $\lambda_{2}$ und addiert, so folgt

$$
-(4 x)\left[\nu_{1}\left(1^{\prime} x\right)+v_{2}\left(2^{\prime} x\right)+v_{3}\left(3^{\prime} x\right)+\left(u_{43} x\right): \lambda_{3}\right]=-v_{3}\left(3^{\prime} x\right)(4 x)-v_{3}^{\prime}(3 x)(4 x),
$$

also

$$
\begin{aligned}
& \left(u_{41} x\right): \lambda_{1}=-v_{2}\left(2^{\prime} x\right)-v_{3}\left(3^{\prime} x\right)+v_{1}(1 x) \\
& \left(u_{42} x\right): \lambda_{2}=-v_{3}\left(3^{\prime} x\right)-v_{1}\left(1^{\prime} x\right)+v_{2}{ }^{\prime}(2 x) \\
& \left(u_{43} x\right): \lambda_{3}=-v_{1}\left(1^{\prime} x\right)-v_{2}\left(2^{\prime} x\right)+v_{3}(3 x)
\end{aligned}
$$

Durch Addition erhält man hieraus und aus (32):

$$
\nu_{1}\left(1^{\prime} x\right)+v_{2}\left(2^{\prime} x\right)+\nu_{3}\left(3^{\prime} x\right)=\nu_{1}^{\prime}(1 x)+\nu_{2}^{\prime}(2 x)+\nu_{3}^{\prime}(3 x)
$$

was übereinstimmt mit (25a), (26a). Daher lassen sich die Gleichungen (33) auch in die Form kleiden:

$$
\begin{aligned}
& \left(u_{41} x\right): \lambda_{1}=-v_{2}^{\prime}(2 x)-v_{3}^{\prime}(3 x)+v_{1}\left(1^{\prime} x\right) \\
& \left(u_{42} x\right): \lambda_{2}=-v_{3}^{\prime}(3 x)-v_{1}^{\prime}(1 x)+v_{2}\left(2^{\prime} x\right) \\
& \left(u_{43} x\right): \lambda_{3}=-v_{1}^{\prime}(1 x)-v_{2}^{\prime}(2 x)+v_{3}\left(3^{\prime} x\right) .
\end{aligned}
$$

Wie man sieht, ist die Konstruktion dev Doppeltangenlen aus einem Aronholdschen System fertig, wenn wir die Tangenten 1', 2', 3' kennen: Denn z. B. ist nach $(26 a) v_{2}\left(2^{\prime} x\right)+v_{3}\left(3^{\prime} x\right)=0$ diejenige Gerade, welche den Punkt $41^{\prime}$ mit dem Punkte $2^{\prime} 3^{\prime}$ verbindet. Ihr Schuittpunkt mit 1 ist nach (33) ein Punkt von $u_{41}$. Einen anderen Punkt von $u_{41}$ erhalte ich nach (33a), wenn ich die, die Punkte 41 und 23 verbindende Gerade zum Schnitt bringe mit $1^{\prime}$. -

Es handelt sich also jetzt darum die Tangenten 1', 2', 3 aus dem Aronholdschen System zu finden.

In (26a) hatten wir eine Darstellung der Geraden 4 durch $1^{\prime}, 2^{\prime}, 3^{\prime}$. Drei entsprechende Darstellungen erhalten wir für 5, 6, 7. Infolgedessen bekommen wir auch noch drei weitere Wertsysteme der Art wie $v_{1}, v_{2}, v_{3}$, die wir daher insgesamt mit $v_{41}, v_{42}, v_{43} ; v_{51}, v_{52}, v_{53}, \ldots, v_{73}$ bezeichnen. Daher 
haben wir jetzt:

$$
\begin{aligned}
& v_{4}(4 x)=v_{41}\left(1^{\prime} x\right)+v_{42}\left(2^{\prime} x\right)+v_{43}\left(3^{\prime} x\right) \\
& v_{5}(5 x)=v_{51}\left(1^{\prime} x\right)+v_{52}\left(2^{\prime} x\right)+v_{53}\left(3^{\prime} x\right) \\
& \left.v_{6} 16 x\right)=v_{61}\left(1^{\prime} x\right)+v_{62}\left(2^{\prime} x\right)+v_{63}\left(3^{\prime} x\right) \\
& v_{7}(7 x)=v_{71}\left(1^{\prime} x\right)+v_{72}\left(2^{\prime} x\right)+v_{73}\left(3^{\prime} x\right)
\end{aligned}
$$

Diese vier Gleichungen sind voneinander abhängig, also verschwindet ihre Determinante :

$$
v_{4}\left(v_{51} v_{61} \nu_{71}\right)(4 x)-v_{5}\left(v_{61} v_{71} \nu_{44}\right)(5 x)+v_{6}\left(v_{71} \nu_{41} \nu_{51}\right)(6 x)-v_{7}\left(v_{41} v_{51} v_{61}\right)(7 x)=0
$$

Andererseits haben wir aber die zwischen vier Geraden der Ebene bestehende Identität $\left(^{1}\right)$ :

$$
(567)(4 x)-(674)(5 x)+(745)(6 x)-(456)(7 x) \equiv 0 .
$$

Daher sind die $\gamma_{i}$ bis auf einen Faktor $g$ durch die Gleichungen bestimmt:

$$
\begin{aligned}
& g \cdot v_{4}=(567):\left(v_{51} v_{61} v_{71}\right) \\
& g \cdot v_{5}=(674):\left(v_{61} v_{71} v_{41}\right) \\
& g \cdot v_{6}=(745):\left(v_{71} v_{41} v_{51}\right) \\
& g \cdot v_{7}=(456):\left(v_{41} v_{51} v_{01}\right) .
\end{aligned}
$$

Danach drücken sich die $1^{\prime}, 2^{\prime}, 3^{\prime}$ folgendermassen durch die $4,5,6$ aus:

$$
\begin{aligned}
& \left(v_{41} \nu_{51} v_{61}\right)\left(1^{\prime} x\right)=v_{4}\left(v_{52} v_{63}-v_{52} v_{53}\right)(4 x)+v_{5}\left(\nu_{43} v_{62}-v_{42} v_{63}\right)(5 x)+ \\
& +v_{6}\left(\nu_{42} \nu_{53}-v_{43} \nu_{52}\right)(6 x) \\
& \left(\nu_{41} \nu_{51} \nu_{61}\right)\left(2^{\prime} x\right)=\nu_{4}\left(\nu_{53} \nu_{61}-\nu_{63} \nu_{51}\right)(4 x)+\nu_{5}\left(\nu_{63} \nu_{41}-\nu_{43} \nu_{61}\right)(5 x)+ \\
& +v_{6}\left(v_{43} v_{51}-v_{53} v_{41}\right)(6 x) \\
& \left(\nu_{41} \nu_{51} \nu_{61}\right)\left(3^{\prime} x\right)=\nu_{4}\left(\nu_{51} \nu_{62}-\nu_{61} \nu_{52}\right)(4 x)+\nu_{5}\left(\nu_{61} \nu_{42}-\nu_{41} \nu_{62}\right)(5 x)+ \\
& +\dot{\nu}_{6}\left(\nu_{41} v_{52}-v_{51} v_{42}\right)(b x) \text {. }
\end{aligned}
$$

Aehnliche Darstellungen erhalten wir, wenn irgend eine andere aus 4, 5, 6, 7 herausgegriffene Basis verwendet wird.

Die einzelnen Determinanten sollen nun genauer untersucht werden.

Nach (26a) ist:

$$
\begin{aligned}
v_{52} v_{63}-v_{62} v_{53} & =(123)^{2}\left[\frac{1}{(531)(612)}-\frac{1}{(631)(512)}\right] \\
& =(123)^{3}(651):(531)(631)(512)(612)
\end{aligned}
$$

(1) Vgl. etwa WEIтzenвöck, Invariantentheorie, 1923, Groningen, § 19. 
(uach der schon bei (37) verwendeten Identität). Entsprechend ist:

$$
v_{43} v_{62}-v_{42} v_{63}=(123)^{\circ}(461):(631)(431)(612)(412) \text { usw. }
$$

$$
\begin{aligned}
\left(v_{41} v_{51} v_{61}\right)= & v_{41}\left(v_{52} v_{63}-v_{53} v_{6 z}\right)+v_{42}\left(v_{53} v_{61}-v_{51} v_{63}\right)+v_{43}\left(v_{54} v_{62}-v_{52} v_{51}\right) \\
= & (123)^{4}[(651)(523)(623)(431)(412)+(652)(531)(631)(412)(423)+ \\
& \quad+(653)(512)(612)(423)(431)]:(412)(423)(431)(512)(523) \\
& (531)(612)(623)(631) \\
= & (123)^{4} Z_{456}: N_{456} .
\end{aligned}
$$

Setzt man diese Werte aus (38), (40) und (41) in (39) ein, so erhält man als erste Gleichung:

$$
\begin{aligned}
g \cdot(123)^{5} Z_{456} \cdot N_{456}^{-1} \cdot\left(1^{\prime} x\right) & =(567)(651)[(531)(631)(512)(612)]^{-1} N_{567} Z_{567}{ }^{-1}(4 x) \\
& +(674)(461)[(631)(431)(612)(412)]^{-1} N_{674} Z_{674}^{-1}(5 x) \\
& +(745)(541)[(431)(531)(412)(512)]^{-1} N_{745} Z_{745}{ }^{-1}(6 x)
\end{aligned}
$$

Es handelt sich jetzt darum, die Natur der Koeffizienten genauer zu bestimmen; mit Ausnahme der $Z$ sind alles dreireihige Determinanten, so dass nur die $Z$ in Frage kommen. Nun ist aber z. B., wenn man das erste der drei Glieder von $Z_{456}$ mittels der schon mehrfach benutztén Identität umformt:

$$
\begin{aligned}
Z_{456}=- & (652)(153)(623)(412)(431)+(653)(152)(623)(412)(431)+ \\
& +(652)(531)(631)(412)(423)+(653)(512)(612)(423)(431) \\
= & (652)(531)(412)[(631)(423)-(623)(431)]+ \\
& \quad+(653)(512)(431)[(612)(423)-(623)(412)] \\
= & (123)[-(652)(531)(412)(643)+(653)(512)(431)(642)] .
\end{aligned}
$$

Das Verschwinden der eckigen Klammer ist aber die Bedingung dafür, dass die 6 Geraden 1, 2,.., 5, 6 auf einem Kegelschnitt liegen ( $\left.{ }^{1}\right)$. Aehnlich mit den anderen $Z$. - Dasselbe Resultat lässt sich aus der Determinante $\left(\nu_{41} v_{51} \nu_{61}\right)$ auch ohne Symbolik ableiten: Es ist nämlich

$$
\left(v_{41} v_{51} v_{61}\right)=(123)^{3} \cdot\left|\begin{array}{ccc}
(423)^{-1} & (523)^{-1} & (623)^{-1} \\
(431)^{-1} & (531)^{-1} & (631)^{-1} \\
(412)^{-1} & (512)^{-1} & (612)^{-1}
\end{array}\right| .
$$

Das Verschwinden der letzteren Determinante bedeutet aber ebenfalls, dass die 6 Geraden, 1,..., 6 auf einem Kegelschnitt liegen ( $\left.{ }^{2}\right) .-$

(1) Vgl. Studx, Einf. in die Theorie der Invarianten, p. 66, oder WEIтzENBöok, Invariantentheorie, $\$ 13(4)$.

(2) Vgl. den Artikel, Two Theorems upon conjugate Conics, im “Tohokn Math. J. », Sept. 1929, wo an diese Determinante erinnert wurde. 
Verschwindet nun $\mathrm{Z}_{456}$, so seigt (42), dass dann 4, 5, 6 durch einen Punkt laufen. Ist aber $Z_{456} \neq 0$, so kam $1^{\prime}$ nach (42) nur dann etwa mit 4 zusammenfallen, wenn 4, 5, 6 durch einen Punkt laufen. - Liefe schliesslich 1' durch den Schnittpunkt von etwa 4 und 5 , so würden, wie sich wiederum aus (42) ergibt, auch irgend drei andere Doppeltangenten des Aronholdschen Systems durch einen Punkt laufen, was aber der Bedingung des Aronholdschen Systems widerspricht.

Damit haben wir exakt den Aronholdschen Satz und seine Umkehrung gewominen:

I. Wenn von einer allgemeinen ebenen Kurve vierter Ordnung ein Aronholdsches System von DT' gegeben ist, dann liegen keine $C$ Tangenten dieses Systems auf einem Kegelschnit.t. Denn dies würde zur Folge haben, dass drei asizygetische Tangenten der Kurve durch einen Punkt laufen, was der Allgemeinheit der Kurve widerspräche. Die übrigen 21 DT lassen sich aus dem Aronholdschen System rational finden.

II. Sind 7 Geraden der Ebene gegeben, von denen keine drei durch einen Punkt laufen und keine 6 auf einem Kegelschnitt liegen, so lassen sich diese immer als ein Aronholdsches System einer Kurve vierter Ordnung auffassen, und es lassen sich aus ihnen die übrigen $21 D T$ und die $C_{4}$ selbst finden. Ist hingegen nur die Bedingung erfüllt, dass keine drei der Geraden des Systems durch einen Punkt laufen, während wohl 6 Geraden des Systems auf einem Kegelschnitt liegen, so bilden die 7 Geraden kein Aronholdsches System einer Kurve vierter Ordnung, da alsdann Gleichung (42) auf der linken Seite verschwinden würde, während ihre rechte Seite nach Voraussetzung immer von Null verschieden ist.

Mit andern Worten: Damit $\%$ Geraden der Ebene ein Aronholdsches System bilden, ist notwendig und hinreichend, dass 1) keine drei von ihnen durch einen Punkt laufen, aber auch 2) dass keine sechs von ihnen auf einem Kegelschnitt liegen. 